Psicologia Escolar

e Educacional
ARTículo

DOI: http://dx.doi.org/1 10.1590/2175-35392021221999

Localizador - e221999

\title{
CLIMA ÁULICO EN LA ESCUELA SECUNDARIA DE LA CIUDAD DE MAR DEL PLATA
}

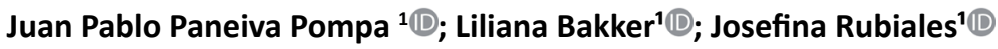

\section{RESUMEN}

El presente trabajo analiza el Clima Áulico en instituciones educativas de nivel secundario de la ciudad de Mar del Plata. La muestra estuvo conformada por 40 grupos Áulicos de nivel secundario básico de cuatro instituciones educativas de la ciudad de Mar del Plata (998 estudiantes, 37 docentes y 39 preceptores). Para evaluar el Clima Áulico se utilizó la Escala de Clima Social Escolar (Classroom Environment Scale, CES) desarrollada por Moos y Tricket. Los resultados señalan un Clima Áulico medianamente favorable. Se presentan las fortalezas y debilidades, así también como las diferencias entre docentes, preceptores y estudiantes. Se resalta la importancia del rol de los preceptores en las instituciones educativas. Se espera que los resultados posibiliten futuras líneas de investigación en el diseño de programas de intervención educativa que favorezcan un Clima Áulico positivo.

Palabras Clave: ambiente de la sala de clase; escuelas secundarias; psicología escolar.

\section{Classroom Climate in the secondary school of Mar del Plata}

\begin{abstract}
The present study analyzes the Classroom Climate in educational institutions of secondary school of the city of Mar del Plata. The sample consisted of 40 classes of basic secondary level of four educational institutions of the city of Mar del Plata (998 students, 37 teachers and 39 preceptors.) To evaluate the Classroom Climate was used the Classroom Environment Scale (CES) developed by Moos and Tricket. The results indicate a moderately favorable Classroom Climate. The strengths and weaknesses are presented, as well as the differences between teachers, preceptors and students. The importance of the role of preceptors in educational institutions is highlighted. The results are expected to enable future lines of research in the design of educational intervention programs that favor a positive Classroom Climate.
\end{abstract}

Keywords: classroom environment; high school; school psychology.

\section{Clima Áulico na escola secundária da cidade de Mar del Plata}

\begin{abstract}
RESUMO
O presente estudo analisa o Clima Áulico em instituições educativas de nível secundário da cidade de Mar del Plata. A mostra foi formada por 40 grupos Áulicos de nível secundário básico de quatro instituições educativas da cidade de Mar del Plata (998 estudantes, 37 docentes e 39 preceptores). Para avaliar o Clima Áulico utilizou-se a Escala de Clima Social Escolar (Classroom Environment Scale, CES) desenvolvida por Moos e Tricket. Os resultados mostram um Clima Áulico medianamente favorável. Apresentam-se os pontos fortes e os pontos fracos, assim também como as diferenças entre docentes, preceptores e estudantes. Ressalta-se a importância do rol dos preceptores nas instituições educativas. Espera-se que os resultados possibilitem futuras linhas de pesquisa no desenho de programas de intervenção educativa que favoreçam um Clima Áulico positivo.
\end{abstract}

Palavras-Chave: ambiente da sala da aula; escolas de ensino médio; psicologia escolar.

\footnotetext{
${ }^{1}$ Instituto de Psicología Básica, Aplicada y Tecnología (IPSIBAT); Facultad de Psicología - Universidad Nacional de Mar del Plata; Consejo Nacional de Investigaciones Científicas y Técnicas (CONICET) - Mar del Plata - Argentina; juampi_paneiva@hotmail.com; lilianabakker@gmail.com; josefinarubiales@gmail.com
} 


\section{INTRODUCCIÓN}

La educación es un proceso complejo en el que convergen los procesos de enseñanza y aprendizaje. Los estudios en educación, dan cuenta de diversas variables que han sido consideradas cruciales en la trasmisión y adquisición de conocimientos, como en el bienestar de los estudiantes y docentes (Bisquerra Alzina, 2008; Carmona \& López, 2015; Paneiva Pompa, Bakker, \& Rubiales, 2018). Las mismas han sido tradicionalmente ubicadas en dos grandes grupos, las que se incluyen dentro de los denominados factores alterables (variables cognitivas/intelectuales, de aptitud para el estudio y afectivas) y aquellas incluidas dentro de los factores que no son susceptibles de ser modificados por el sistema educativo, denominados inalterables (posición socioeconómica, nivel educativo de los padres, tamaño de la familia, entre otras) (Chávez Uribe, 2006). Sin embargo, como señalan Sternberg, Wagner, Williams y Horvath (1995) estos factores son pobres predictores del rendimiento académico a largo plazo y del éxito laboral fuera de los entornos educativos. Por consiguiente, diversas investigaciones están orientadas hacia la búsqueda de factores no cognitivos que permitan un mayor conocimiento de las causas y factores que influyen en problemáticas actuales y frecuentes en los ámbitos educativos, así como en el diseño de programas para mejorar el proceso de aprendizaje de los alumnos (Bisquerra Alzina, 2008). En esta área de escaso estudio se encuentra el Clima Áulico. Coll y Solé (2004) señalan que hasta hace algunas décadas el contexto del aula ha estado prácticamente ausente en las investigaciones sobre el aprendizaje en la escuela. Sin embargo, en la actualidad, el panorama ha ido cambiando, y la investigación sobre las características del contexto de enseñanza y de aprendizaje va alcanzando mayor protagonismo (Treviño et al., 2010).

El Clima Áulico puede ser definido como el entorno intelectual, social, emocional y físico en el que los estudiantes aprenden (Ambrose, Bridges, DiPietro, \& Lovett, 2010). El mismo es un reflejo de las opiniones de los estudiantes sobre su experiencia en el contexto académico (Reid \& Radhakrishnan, 2003). Diversos trabajos señalan que un Clima Áulico positivo favorece el desarrollo cognitivo y emocional de los estudiantes, beneficiando la adquisición de habilidades cognitivas, el aprendizaje, el desarrollo de actitudes positivas hacia el estudio y el rendimiento académico (Cornejo, \& Redondo, 2001; Norton, 2008; Pianta, La Paro, Payne, Cox, \& Bradley, 2002; Triano Quijano, \& Velázquez Niño, 2014).

Considerando la necesidad de contar con mayor evidencia empírica que valide la implementación de estrategias que favorezcan un Clima Áulico positivo, este estudio tuvo como objetivo evaluar y analizar los niveles de Clima Áulico en clases de secundaria básica de instituciones educativas de la ciudad de Mar del
Plata. Por lo tanto, se describen los niveles de Clima Áulico de los grupos Áulicos en términos de Relaciones, Desarrollo Personal, Estabilidad y Sistema de Cambio. A su vez, se comparan las percepciones de estudiantes, docentes y preceptores. En este sentido, se busca identificar los elementos de la clase que intervienen de manera sustancial en el proceso educativo, y se espera que los resultados posibiliten el diseño de programas de intervención educativa que favorezcan un Clima Áulico positivo.

\section{METOdOLOGÍA}

\section{Instrumento}

Para evaluar el Clima Áulico se utilizó la Escala de Clima Social Escolar (Classroom Environment Scale, CES) desarrollada por Moos y Tricket (1974). La misma se focaliza en los aspectos socioemocionales-ambientales de la clase, los cuales hacen referencia a la dinámica social del sistema que incluye no sólo el comportamiento del docente, sino también la relación docente-estudiantes y la de los estudiantes entre sí.

Para la presente investigación se administró la versión adaptada al español-castellano (Casullo \& Mikulic, 2009), a la cual se le incorporó una forma de respuesta del tipo Likert con cuatro opciones: "Nunca", "A veces", "Casi siempre" y "Siempre", debiendo marcar con una " $X$ " la opción que consideraban correcta. Tanto la versión original como la versión adaptada, se componen de 90 ítems agrupados en 9 subescalas comprendidas en cuatro dimensiones: Relacional, Desarrollo Personal, Estabilidad y Sistema de Cambio.

A continuación se presenta un breve cuadro en donde se describen las nueve subescalas del CES y se detalla a qué dimensión pertenecen.

\section{Población y muestra}

El universo del estudio corresponde a grupos áulicos pertenecientes al ciclo básico del nivel de educación secundaria de la ciudad de Mar del Plata. La muestra estuvo conformada por 40 grupos Áulicos de nivel secundario básico [12 de primer año (30\%), 14 de segundo año (35\%) y 14 de tercer año (35\%)], de cuatro (4) instituciones educativas de la ciudad de Mar del Plata. El total de la muestra estuvo conformada por 998 estudiantes, 37 docentes y 39 preceptores.

\section{Procedimiento}

Se realizaron los contactos con cuatro instituciones educativas de la ciudad de Mar del Plata, provincia de Buenos Aires, Argentina. Las sesiones de evaluación con los cursos se efectuaron en forma grupal y autoadministrada. La participación fue voluntaria y sujeta al consentimiento informado de los padres, docentes y directivos; y el asentimiento de los estudiantes participantes. Previo a la administración del instrumento se procedió 
Cuadro 1. Dimensiones y subescalas del CES (Moos \& Tricket, 1974).

\begin{tabular}{|c|c|c|c|}
\hline Dimensión & Definición & Sub-escalas & Definición \\
\hline \multirow{3}{*}{ Relacional } & \multirow{3}{*}{$\begin{array}{l}\text { Grado de implicación de los } \\
\text { estudiantes en el ambiente, alcance } \\
\text { de su apoyo y ayuda hacia el otro } \\
\text { y grado de libertad de expresión. } \\
\text { Es decir mide qué tan integrados } \\
\text { están los estudiantes en la clase, se } \\
\text { apoyan y ayudan entre sí. }\end{array}$} & Implicación & $\begin{array}{c}\text { Grado en que los alumnos muestran interés por } \\
\text { las actividades de la clase y participan en los } \\
\text { coloquios y cómo disfrutan del ambiente creado, } \\
\text { incorporando tareas complementarias. }\end{array}$ \\
\hline & & Afiliación & $\begin{array}{l}\text { Nivel de amistad entre los alumnos y cómo se } \\
\text { ayudan en sus tareas, se conocen y disfrutan } \\
\text { trabajando juntos. }\end{array}$ \\
\hline & & Ayuda & $\begin{array}{l}\text { Grado de ayuda, preocupación y amistad por los } \\
\text { alumnos (comunicación abierta con los alumnos, } \\
\text { confianza en ellos e interés por sus ideas). }\end{array}$ \\
\hline \multirow{2}{*}{$\begin{array}{l}\text { Desarrollo } \\
\text { Personal }\end{array}$} & \multirow{2}{*}{$\begin{array}{l}\text { Importancia que se concede en la } \\
\text { clase a la realización de las tareas y } \\
\text { a los temas de las materias. }\end{array}$} & Tarea & $\begin{array}{l}\text { Importancia que se la da a la terminación de las } \\
\text { tareas programadas. Énfasis que pone el profesor } \\
\text { en el temario de las materias. }\end{array}$ \\
\hline & & Competitividad & $\begin{array}{l}\text { Grado de importancia que se da al esfuerzo por } \\
\text { lograr una buena calificación y estima, así como a } \\
\text { la dificultad para obtenerlas. }\end{array}$ \\
\hline \multirow{3}{*}{$\begin{array}{c}\text { Sistema de } \\
\text { Mantenimiento }\end{array}$} & \multirow{3}{*}{$\begin{array}{l}\text { Evalúa las actividades relativas } \\
\text { al cumplimiento de objetivos, } \\
\text { funcionamiento adecuado de la } \\
\text { clase, organización, claridad y } \\
\text { coherencia en la misma. }\end{array}$} & Organización & $\begin{array}{l}\text { Importancia que se le da al orden, organización } \\
\text { y buenas maneras en la realización de las tareas } \\
\text { escolares. }\end{array}$ \\
\hline & & Claridad & $\begin{array}{l}\text { Importancia que se da al establecimiento y } \\
\text { seguimiento de normas claras y al conocimiento } \\
\text { por parte de los alumnos de las consecuencias de } \\
\text { su incumplimiento. } \\
\text { Grado en que el profesor es coherente con esa } \\
\text { normativa e incumplimientos. }\end{array}$ \\
\hline & & Control & $\begin{array}{l}\text { Grado en que el profesor es estricto en sus } \\
\text { controles sobre el cumplimiento de las normas y } \\
\text { penalización de aquellos que no las practican. }\end{array}$ \\
\hline $\begin{array}{l}\text { Sistema de } \\
\text { Cambio }\end{array}$ & $\begin{array}{l}\text { Grado en que existen diversidad, } \\
\text { novedad y variación razonables en } \\
\text { las actividades de clase. }\end{array}$ & Innovación & $\begin{array}{l}\text { Grado en que los alumnos contribuyen a planear } \\
\text { las actividades escolares y la variedad y cambios } \\
\text { que introduce el profesor con nuevas técnicas y } \\
\text { estímulos a la creatividad del alumno. }\end{array}$ \\
\hline
\end{tabular}

a la lectura de las instrucciones de la prueba. Durante el desarrollo del trabajo se respetaron los principios éticos de la investigación con seres humanos, procurándose las condiciones necesarias para proteger la confidencialidad y actuar en beneficio de los participantes.

\section{Análisis de datos}

Para realizar los análisis estadísticos se utilizó el paquete SPSS (Statistical Package for the Social Sciences) versión 19. Los puntajes brutos obtenidos fueron transformados a partir de una escala de 0 a 3 puntos de acuerdo a las opciones de respuesta utilizadas, adaptando el baremo de Rivera Arrieta (2018) de cinco intervalos de interpretación: 0 a 0,6 = muy desfavorable; 0,7 a 1,2 = desfavorable; 1,3 a 1,8 medianamente favorable; 1,9 a 2,4 = altamente favorable; y 2,5 a 3 = altamente favorable. El puntaje para Clima Áulico total se obtuvo de la media de las 4 dimensiones evaluadas. Con el objeto de describir los niveles de Clima Áulico los datos obtenidos fueron sometidos a un análisis estadístico descriptivo.

Debido a que los datos no cumplen con la hipótesis de homocestacidad (Pardo \& Ruiz, 2000), se aplicaron las pruebas $U$ de Mann-Whitney y Kruskal-Wallis para comparar medias y para establecer si las diferencias observadas son estadísticamente significativas. Adicionalmente, se calculó el tamaño del efecto de la diferencia, para lo cual se utilizó la clasificación por rangos de Cohen, que indica que el tamaño del efecto puede ser grande (mayor a 0,8), mediano (cercano a 0,5) o menor (menor a 0,2) (Quezada, 2007). 


\section{RESULTADOS}

\section{Clima Áulico total}

En relación con la descripción del Clima Áulico en la escuela secundaria de la ciudad de Mar del Plata, los resultados indican una mediana de 1,45 puntos sobre 3 , que al ser comparado con el baremo de interpretación indica un Clima medianamente favorable. El puntaje mínimo fue de 0,55 y el máximo de 1,99; siendo el desvío típico de 0,22 . En el mismo sentido, podemos observar en el gráfico 1 que el $75 \%$ de los datos puntúa por encima 1,31; lo que de acuerdo al baremo refiere un Clima medianamente favorable.

Se observa en el gráfico 1 una distribución homogénea y simétrica, lo que indicaría poca variabilidad y una distribución de frecuencias similar por encima y debajo de la mediana. Del mismo modo, se observa un puntaje mínimo de 0,55 y un puntaje máximo de 1,99; y la existencia de algunos valores atípicos, es decir alejados de la distribución normal de los datos, por debajo del primer cuartil.

\section{Dimensiones de Clima Áulico}

En la Tabla 1 se presentan los resultados para las dimensiones de Clima Áulico en la escuela secundaria de la Ciudad de Mar del Plata. La misma refleja que todas las dimensiones se ubican en la categoría medianamente favorable. Sin embargo, la dimensión que presentó una mediana menor fue Sistema de Cambio con un puntaje de 1,37; y la de mejor puntuación la dimensión Desarrollo Personal con una mediana de 1,60.

En el gráfico 2 se observa que todas las dimensiones presentaron una distribución simétrica lo que indicaría una distribución de frecuencias similar, por encima y debajo de la mediana. A su vez, la dimensión Desarrollo Personal presentó la distribución más homogénea, mientras que Sistema de Cambio fue la que presentó mayor heterogeneidad. Todas las dimensiones presentan casos atípicos.

\section{Sub-escalas de Clima Áulico}

En la Tabla 2 se presentan los resultados para las sub-escalas de Clima Áulico en la escuela secundaria de la Ciudad de Mar del Plata. La misma refleja que las sub-escalas implicación y control presentan las medianas más bajas, con un puntaje de 1,18; las cuales de acuerdo a las categorías de interpretación resultan desfavorables. Las demás sub-escalas presentaron puntajes que según la escala de interpretación son considerados medianamente favorables, a excepción de la sub-escala claridad con una mediana de 1,82 considerada altamente favorable.

En el Gráfico 3 se representa la distribución de frecuencias de los puntajes de las sub-escalas de Clima Áulico. En el mismo se puede observar cajas alargadas, lo que indica mayor heterogeneidad en los datos con respecto al Clima Áulico total, es decir presentan características dispersas y mayor variabilidad.

Diferencias entre las percepciones de docentes, preceptores y estudiantes

En la Tabla 3 se presentan los resultados para las dimensiones y sub-escalas de Clima Áulico en la escuela secundaria de la ciudad de Mar del Plata según estudian-

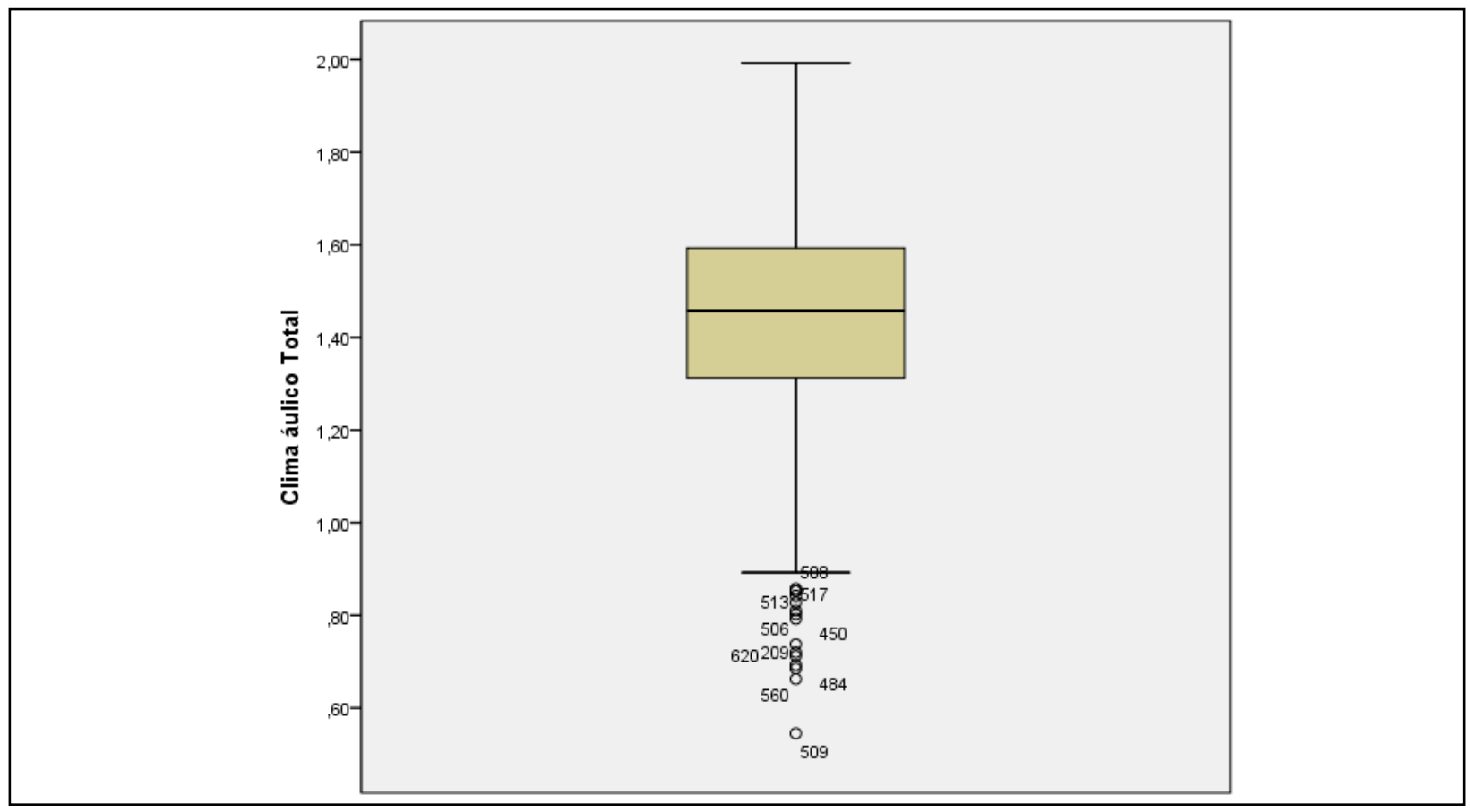

Gráfico 1. Diagrama de cajas y bigotes de Clima Áulico total. 
Tabla 1. Estadísticos Dimensiones de Clima Áulico en la Escuela Secundaria de la ciudad de Mar del Plata.

\begin{tabular}{|c|c|c|c|c|c|}
\hline & & Relacional & Desarrollo Personal & $\begin{array}{c}\text { Sistema de } \\
\text { Mantenimiento }\end{array}$ & Sistema de Cambio \\
\hline \multicolumn{2}{|c|}{ Media } & 1,41 & 1,59 & 1,39 & 1,37 \\
\hline \multicolumn{2}{|c|}{ Mediana } & 1,42 & 1,60 & 1,39 & 1,36 \\
\hline \multicolumn{2}{|c|}{ Desvío típico } & ,37 & ,28 & 31 & ,40 \\
\hline \multicolumn{2}{|c|}{ Mínimo } & ,00 & , 00 & ,00 & ,00 \\
\hline \multicolumn{2}{|c|}{ Máximo } & 2,48 & 2,34 & 2,27 & 2,64 \\
\hline \multirow{3}{*}{ Percentiles } & 25 & 1,18 & 1,45 & 1,21 & 1,09 \\
\hline & 50 & 1,42 & 1,60 & 1,39 & 1,36 \\
\hline & 75 & 1,67 & 1,77 & 1,61 & 1,64 \\
\hline
\end{tabular}

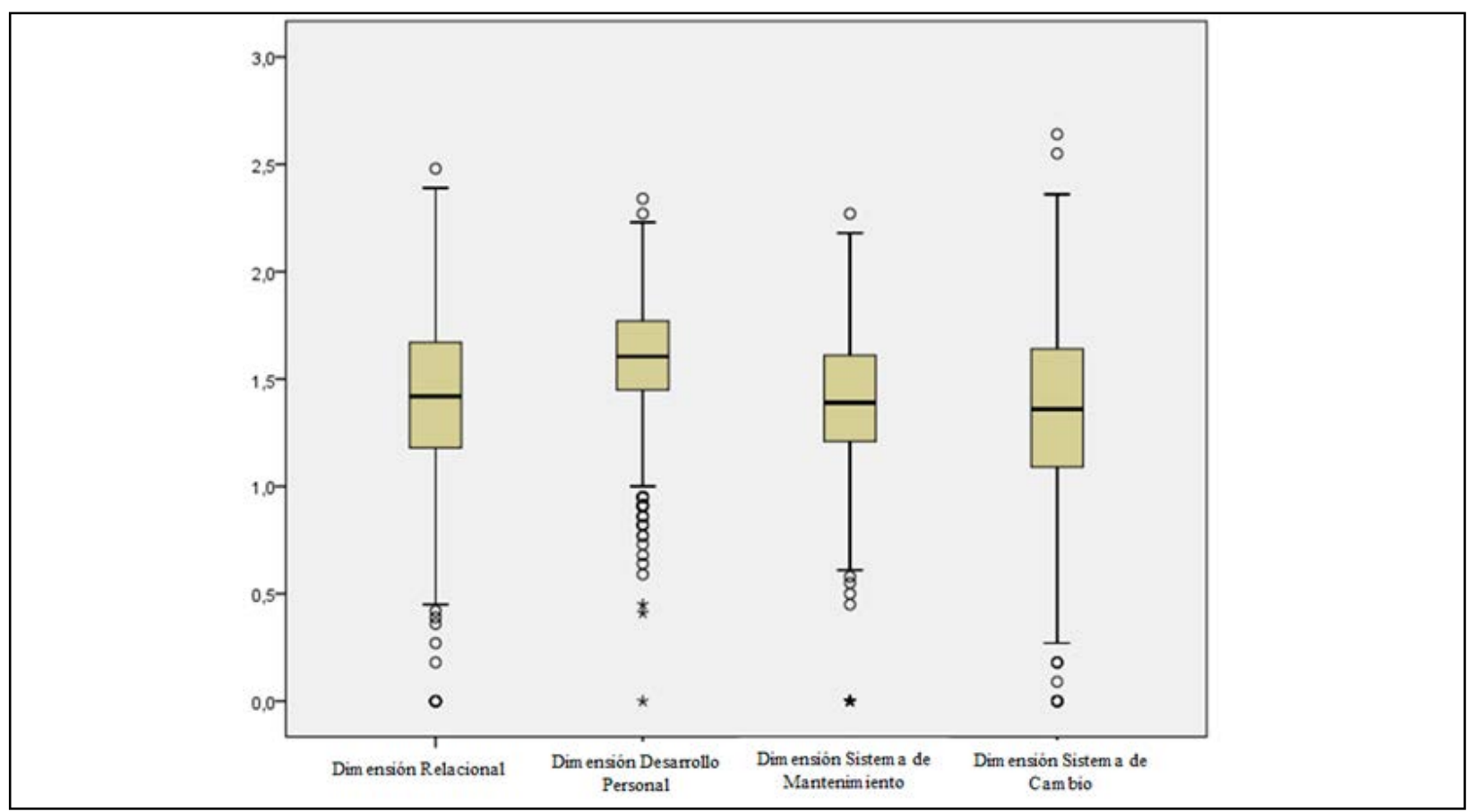

Gráfico 2. Diagrama de cajas y bigotes de las dimensiones de Clima Áulico.

tes, docentes y preceptores. Se encontraron diferencias significativas en las dimensiones Relacional, Desarrollo Personal y Sistema de Cambio, como a su vez en todas las sub-escalas a excepción de tarea e innovación. En las sub-escalas competitividad y control las diferencias fueron menores a 0,05; mientras que en implicación, afiliación, ayuda, organización y claridad las diferencias fueron menores a 0,01.

A continuación se presenta la tabla 4 en la que se muestran los resultados para las dimensiones y sub-escalas de Clima Áulico en la escuela secundaria de la ciudad de Mar del Plata comparando docentes con estudiantes, docentes con preceptores y preceptores con estudiantes.

Al comparar docentes con estudiantes, se encontraron diferencias estadísticamente significativas en todas las dimensiones y sub-escalas a excepción de Tarea y Desarrollo Personal. Las diferencias fueron a favor de los docentes con un tamaño del efecto grande en las sub-escalas implicación, afiliación, ayuda, claridad y la dimensión Relacional; y un tamaño del efecto mediano en organización, innovación y la dimensión Sistema de Mantenimiento. Los estudiantes presentaron puntajes superiores estadísticamente significativos en las sub-escalas control y competitividad con un tamaño del efecto mediando. Sin embargo, al comparar docentes con preceptores sólo se encontraron diferencias estadísticamente significativas en la sub-escala ayuda y la dimensión Relacional, con un tamaño del efecto mediano.

Por otro lado, al comparar preceptores con estudiantes se encontraron diferencias estadísticamente significativas en las sub-escalas implicación, afiliación, 
Tabla 2. Estadísticos Sub-escalas de Clima Áulico en la Escuela Secundaria de la ciudad de Mar del Plata.

\begin{tabular}{|c|c|c|c|c|c|c|c|c|c|c|}
\hline & & Implicación & Afiliación & Ayuda & Tarea & Competitividad & Organización & Claridad & Control & Innovación \\
\hline \multicolumn{2}{|c|}{ Media } & 1,16 & 1,47 & 1,61 & 1,63 & 1,56 & 1,26 & 1,76 & 1,17 & 1,37 \\
\hline \multicolumn{2}{|c|}{ Mediana } & 1,18 & 1,45 & 1,64 & 1,64 & 1,55 & 1,27 & 1,82 & 1,18 & 1,36 \\
\hline \multicolumn{2}{|c|}{ Desvío típico } & ,44 & ,45 & ,46 & ,33 & ,35 & ,39 & ,40 & ,40 & ,40 \\
\hline \multicolumn{2}{|c|}{ Mínimo } & ,00 & ,00 & ,00 &, 55 & ,00 & ,00 & ,55 & ,00 & ,00 \\
\hline \multicolumn{2}{|c|}{ Máximo } & 2,45 & 2,73 & 2,64 & 2,64 & 2,55 & 2,36 & 2,73 & 2,36 & 2,64 \\
\hline \multirow{3}{*}{ Percentiles } & 25 & ,82 & 1,18 & 1,27 & 1,36 & 1,36 & 1,00 & 1,55 & ,91 & 1,09 \\
\hline & 50 & 1,18 & 1,45 & 1,64 & 1,64 & 1,55 & 1,27 & 1,82 & 1,18 & 1,36 \\
\hline & 75 & 1,45 & 1,80 & 1,91 & 1,91 & 1,82 & 1,55 & 2,09 & 1,45 & 1,64 \\
\hline
\end{tabular}

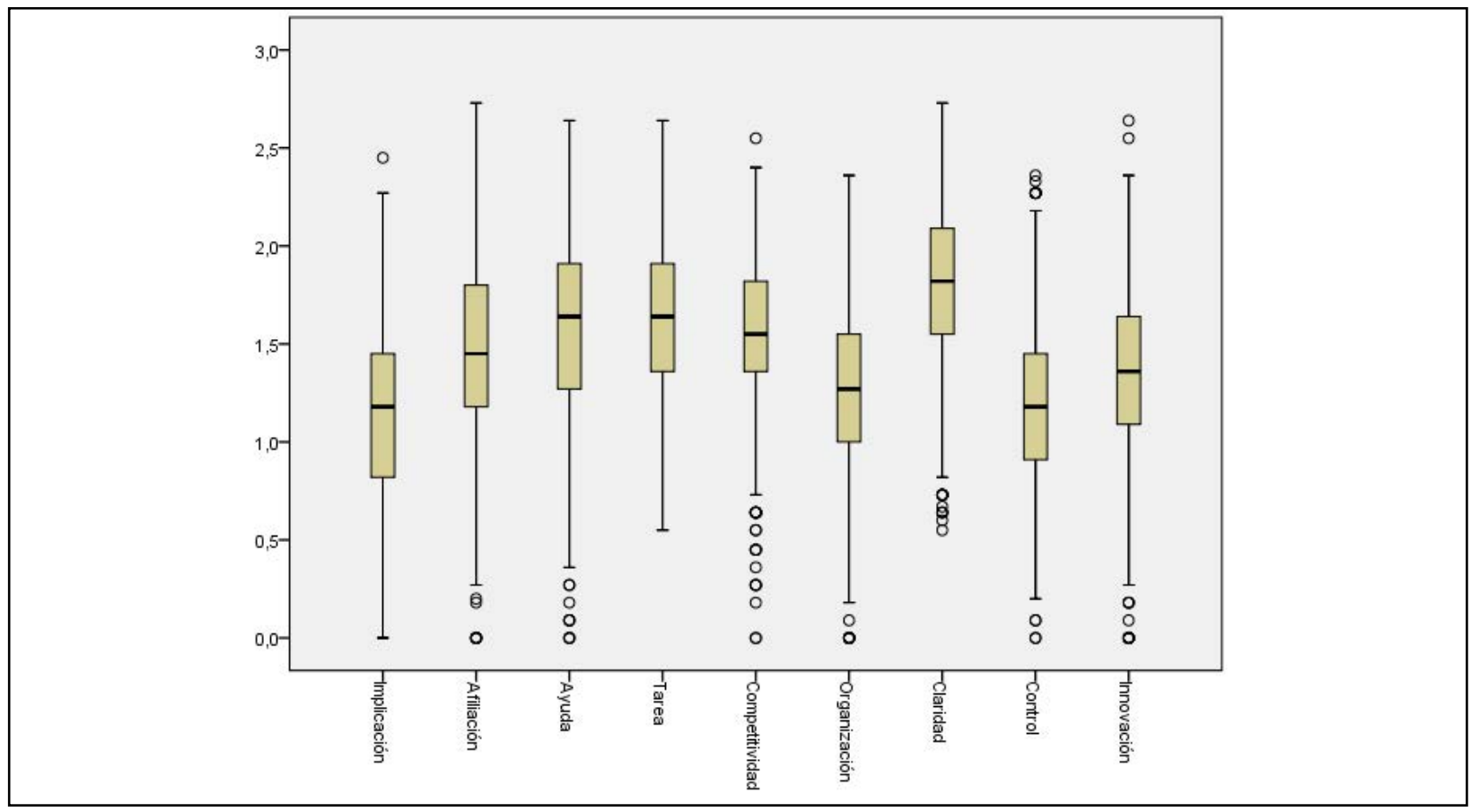

Gráfico 3. Diagrama de cajas y bigotes de Sub-escalas de Clima Áulico.

organización y claridad, y en las dimensiones Relacional y Sistema de Mantenimiento. Las diferencias resultaron con un tamaño del efecto mediano en las sub-escalas afiliación y claridad y en la dimensión Sistema de Mantenimiento; y un tamaño del efecto grande en las sub-escalas implicación y organización y en la dimensión Relacional.

\section{DISCUSIÓN}

Al evaluar y analizar los niveles de Clima Áulico en nivel secundario básico de la ciudad de Mar del Plata, los hallazgos señalan que presenta niveles medianamente favorables, lo cual como se ha señalado sería beneficioso para la adquisición de habilidades cognitivas, el aprendizaje, el desarrollo de actitudes positivas hacia el estudio y el rendimiento académico (Cornejo \& Redondo, 2001; Norton, 2008; Pianta et al., 2002;
Triano Quijano \& Velázquez Niño, 2014). Estos resultados se encuentran en sintonía con los hallazgos de las pruebas APRENDER, una evaluación realizada por el Ministerio de Educación de la Nación, que tiene como objetivo obtener datos sobre el estado de la educación Argentina para planificar políticas públicas educativas. En la misma, los estudiantes secundarios de la ciudad de Mar del Plata obtuvieron resultados superiores en sus aprendizajes al promedio de la provincia de Buenos Aires y del país (Ministerio de Educación de la Nación, 2018). Es decir, los niveles medianamente favorables de Clima Áulico hallados en este estudio pueden ser un factor que esté incidiendo positivamente en los resultados académicos de los alumnos.

En cuanto al análisis de los resultados según dimensiones, los mismos indican que Sistema de Cambio presentó el puntaje menor, indicando como debilidad 
Tabla 3. Estadísticos de las dimensiones y sub-escalas de Clima Áulico en la Escuela Secundaria de la ciudad de Mar del Plata y resultados de la prueba Kruskall-Wallis discriminados según estudiantes, docentes y preceptores.

\begin{tabular}{|c|c|c|c|c|c|c|c|c|c|}
\hline \multirow{2}{*}{ Dimensión } & \multirow{2}{*}{ Sub-escala } & \multicolumn{2}{|c|}{ Estudiantes } & \multicolumn{2}{|c|}{ Docentes } & \multicolumn{2}{|c|}{ Preceptores } & \multicolumn{2}{|c|}{ Estadísticos de contraste } \\
\hline & & M & $\mathrm{DE}$ & M & $\mathrm{DE}$ & M & $\mathrm{DE}$ & $x^{2}$ & $\mathbf{p}$ \\
\hline \multirow{4}{*}{ Relacional } & Implicación & 1,13 & ,43 & 1,57 &, 40 & 1,53 & ,37 & 61,52 &, $00 * *$ \\
\hline & Afiliación & 1,44 & ,44 & 1,92 & ,50 & 1,74 & ,39 & 45,31 &, $00 * *$ \\
\hline & Ayuda & 1,60 & ,45 & 1,98 & ,35 & 1,73 & ,43 & 28,96 &, $00 * *$ \\
\hline & Total & 1,38 & ,36 & 1,83 & ,34 & 1,67 & ,31 & 66,02 &, $00 * *$ \\
\hline \multirow{3}{*}{ Desarrollo Personal } & Tarea & 1,63 &, 34 & 1,67 & 27 & 1,65 & ,30 & ,983 & ,61 \\
\hline & Competitividad & 1,56 & ,36 & 1,43 & ,28 & 1,49 &, 27 & 8,57 &, $01 *$ \\
\hline & Total & 1,59 &, 29 & 1,55 & 19 & 1,57 & 23 & 2,31 & $00 * *$ \\
\hline \multirow{4}{*}{ Mantenimiento } & Organización & 1,24 & ,39 & 1,45 & ,28 & 1,56 & ,32 & 36,03 &, $00 * *$ \\
\hline & Claridad & 1,74 & ,39 & 2,15 & ,28 & 1,98 & ,43 & 50,30 &, $00 * *$ \\
\hline & Control & 1,18 & ,41 & 1,03 & 27 & 1,16 & ,33 & 6,41 &, $04 *$ \\
\hline & Total & 1,38 & ,31 & 1,55 & ,22 & 1,57 & ,29 & 27,78 &, $00 * *$ \\
\hline Sistema de Cambio & Innovación & 1,36 & 40 & 1,57 &, 45 & 1,34 &, 29 & 9,86 & ,07 \\
\hline
\end{tabular}

$*: p<, 05 . ; *: p<, 01$. Ref.: M (Media), DE (Desvío Típico).

Tabla 4. Resultados de la prueba U-Mann-Whitney, significación asintótica bilateral y d’ de Cohen comparando docentes con estudiantes, docentes con preceptores y preceptores con estudiantes.

\begin{tabular}{|c|c|c|c|c|c|c|c|}
\hline \multirow{2}{*}{ Dimensión } & \multirow{2}{*}{ Sub-escala } & \multicolumn{2}{|c|}{ Docentes vs estudiantes } & \multicolumn{2}{|c|}{ Docentes vs preceptores } & \multicolumn{2}{|c|}{ Preceptores vs estudiantes } \\
\hline & & p & $d^{\prime}$ & $p$ & $d^{\prime}$ & $p$ & $d^{\prime}$ \\
\hline \multirow{4}{*}{ Relacional } & Implicación &, $00 * *$ & 1,02 & ,70 & - &, $00 * *$ & ,93 \\
\hline & Afiliación &, $00 * *$ & 1,08 & 12 & - &, $00 * *$ & ,68 \\
\hline & Ayuda &, $00 * *$ & ,85 &, $00 * *$ & ,63 & 07 & - \\
\hline & Total & $00 * *$ & 1,25 &, $04 *$ &, 49 &, $00 * *$ &, 80 \\
\hline \multirow{3}{*}{ Desarrollo Personal } & Tarea & ,38 & - & ,69 & - & 62 & - \\
\hline & Competitividad &, $01^{*}$ & ,36 & ,30 & - & 13 & - \\
\hline & Total & 17 & - &, 55 & - & ,47 & - \\
\hline \multirow{4}{*}{$\begin{array}{c}\text { Sistema de } \\
\text { Mantenimiento }\end{array}$} & Organización &, $00 * *$ &, 54 & ,09 & - &, $00 * *$ & ,82 \\
\hline & Claridad &, $00 * *$ & 1,06 & 10 & - &, $00 * *$ & 61 \\
\hline & Control &, $01 *$ & ,37 & ,05 & - & ,70 & - \\
\hline & Total &, $00 * *$ &, 55 & ,28 & - &, $00 * *$ & 61 \\
\hline Sistema de Cambio & Innovación &, $00 * *$ &, 52 &, $00 * *$ & ,61 & ,68 & - \\
\hline
\end{tabular}

$*: p<, 05 .{ }^{* *}: p<, 01$.

el grado en que existen diversidad, novedad y variación razonable en las actividades de clase. Por otro lado, la mejor puntuación la presentó la dimensión Desarrollo Personal señalando la importancia que se le concede en las clases a la realización de las tareas y a los temas de las materias.

En cuanto a las sub-escalas los resultados mostraron que los puntajes más bajos se encontraron en implicación y control, lo que respectivamente indicaría un bajo interés por las actividades de la clase, poca participación en las actividades grupales y disfrute del ambiente creado incorporando tareas complementarias; con una fle- xibilidad excesiva del profesor en sus controles sobre el cumplimiento de las normas y penalización de aquellos que no las practican. Por otro lado, los puntajes más altos se encontraron en la sub-escala claridad, indicando que se le brinda mucha importancia al establecimiento y seguimiento de normas claras y al conocimiento por parte de los alumnos de las consecuencias de su incumplimiento. En síntesis, estos datos nos permitirían pensar que en la escuela secundaria de la ciudad de Mar del Plata existiría claridad para explicar y explicitar las normas de una clase, pero un bajo grado de coherencia con las normativas y sus incumplimientos. 
Al comparar docentes con estudiantes, los hallazgos mostraron diferencias significativas en favor de los estudiantes, quienes obtuvieron puntajes superiores en las sub-escalas competitividad y control, lo que indicaría mayor percepción del grado de importancia que se le asigna al esfuerzo por lograr buenas calificaciones y la dificultad percibida para obtenerlas, y un mayor nivel de rigurosidad por parte del docente sobre el cumplimiento de las normas y las consecuencias sobre quienes no las cumplen. Por otro lado, los resultados mostraron que fueron los docentes quienes puntuaron significativamente superior en las sub-escalas implicación, afiliación, ayuda, organización y claridad; en las dimensiones Relacional, Mantenimiento del Sistema y Sistema de Cambio. Lo que indicaría que los docentes perciben que sus estudiantes muestran mayor interés por las actividades de la clase de lo que realmente ellos prestan y un mayor grado de compromiso, cooperación entre ellos e integración del que realmente muestran. A su vez, los docentes se percibirían con un vínculo más estrecho con sus alumnos del que ellos perciben, basado en una mayor preocupación por sus inquietudes, la creencia en una comunicación más abierta y toma de consideración en las contribuciones que les realizarían en la planeación de las actividades escolares. Además, los docentes sostendrían que en sus clases habría un mayor cumplimiento de los objetivos propuestos, orden, organización y coherencia en el cumplimiento de las normas del que sus estudiantes perciben.

Por otro lado, al comparar docentes con preceptores, es de resaltar la similitud en la percepción del Clima del aula. Si bien los preceptores no comparten las clases, y muchas veces su conocimiento del Clima Áulico es a través de la deducción o suposición, los hallazgos señalan que no habría diferencias significativas excepto en la sub-escala ayuda y las dimensiones Relacional y Sistema de Cambio. En estas variables son los docentes quienes se percibirían con mayor preocupación y comunicación con sus alumnos, y en definitiva mejor vínculo del que sus pares preceptores percibirían.

Finalmente al comparar los resultados entre estudiantes y preceptores, los mismos muestran que todas las diferencias significativas fueron en favor de los preceptores. En lo que atañe a las sub-escalas implicación, afiliación, organización y claridad, y las dimensiones Relacional y Sistema de Mantenimiento, los resultados señalan que al igual que al comparar con los docentes, los estudiantes puntúan significativamente menos. Lo que significaría que tanto los niveles de cooperación y ayuda mutua, el interés por las actividades de clase, como la importancia que se les da al orden, organización y buenas maneras en la realización de las tareas escolares, como al establecimiento y seguimiento de normas claras es inferior al que los adultos perciben.

\section{CONCLUSIONES}

Al evaluar y analizar los niveles de Clima Áulico en la escuela secundaria básico de la ciudad de Mar del Plata, los hallazgos señalan que presenta como fortaleza la importancia que se le brinda al establecimiento, seguimiento y al conocimiento por parte de los estudiantes de las normas y las consecuencias de su incumplimiento, la terminación de las tareas programadas; el grado de ayuda, preocupación y vínculo que los docentes presentan con los estudiantes; y la importancia que se da al esfuerzo por lograr una buena calificación y estima por parte del docente. Por el contrario, presenta como debilidad la menor importancia dada al orden de la clase y al cumplimiento de las normas establecidas, concluyendo que si bien las normas de la clase son claras y conocidas, no existiría coherencia con la exigencia en su observancia y las consecuencias ante su incumplimiento. En este sentido, un punto a trabajar y continuar evaluando es la adecuación entre normas y consecuencias, ya sea porque las primeras sean exageradas o porque no existe una adecuada implementación de las segundas. Por otro lado, los resultados señalaron que si bien todas las dimensiones presentaron nivelen medianamente favorables, aquella que mide el Sistema de Cambio presentó los resultados más bajos. Lo mismo nos permite proponer que uno de los aspectos por fortalecer es la introducción de diversidad, novedad y variaciones razonables en las actividades de clase. En este sentido, podría señalarse para futuras investigaciones la evaluación de cambios que se introducen en las clases y cuáles son aquellos estímulos a la creatividad más valorados por los estudiantes.

Al comparar la percepción del Clima Áulico entre docentes, preceptores y estudiantes, podemos concluir que existen percepciones similares entre docentes y preceptores. Lo mismo podría resultar un elemento de gran importancia para los equipos directivos de las instituciones escolares, ya que los preceptores forman parte del personal que se encuentra mayor cantidad de tiempo en la institución, y se podría contar con ellos como informantes clave de las características del Clima Áulico de las diferentes clases. Por otro lado, podemos señalar que los docentes perciben un nivel de Clima Áulico mejor que el percibido por los estudiantes, lo cual podría explicarse a la existencia de cierta deseabilidad social en las respuestas de los docentes, que podrían atribuirse a las expectativas por formar parte de la investigación y mostrar su tarea de manera satisfactoria.

Los resultados obtenidos, resaltan la importancia del rol de los preceptores en las instituciones educativas, como así también señalan la importancia de continuar trabajando en la coherencia entre las normas establecidas y su cumplimiento. 
Finalmente se espera que los hallazgos posibiliten futuras líneas de investigación en el diseño de programas de intervención educativa que favorezcan un Clima Áulico positivo, y en consecuencia permitan mejorar el proceso de aprendizaje y el bienestar de alumnos y docentes.

\section{REFERENCIAS}

Ambrose, S. A.; Bridges, M. W.; DiPietro, M.; Lovett, M. C. (2010). How learning works: Seven research-based principles for smart teaching. San Francisco, CA: Jossey Bass.

Bisquerra Alzina, R. (2008). Educación emocional y bienestar. Universidad de Barcelona. Madrid: Wolters Kluwer España.

Carmona, M. G.; López, J. E. (2015). Autoconcepto, dificultades interpersonales, habilidades sociales y conductas asertivas en adolescentes. Revista Española de Orientación y Psicopedagogía, 26(2), 42-58.

Cassullo, G.; Mikulic, I. (2009). Algunas consideraciones acerca del Concepto de clima social y su evaluación. Universidad de Buenos Aires. Recuperado en 01 mar. 2017, de http://23118.psi.uba.ar/academica/carrerasdegrado/ psicologia/informacion_adicional/obligatorias/059_ psicometricas1/tecnicas_psicometricas/archivos/ficha_4. pdf

Chávez Uribe, A. (2006). Bienestar psicológico y su influencia en el rendimiento académico de estudiantes de nivel medio superior. Tesis de maestría, Facultad de Psicología, Universidad de Colima, Colima. Recuperado de http:// digeset.ucol.mx/tesis_posgrado/Pdf/ALFONSO_CHAVEZ_ URIBE.pdf

Coll, C.; Solé, I. (2004). Enseñar y aprender en el contexto del aula. In Coll, C.; Marchesi, A.; Palacios, J. (Eds.), Desarrollo psicológico y educación. Psicología de la educación escolar (Vol. 2, pp. 357-386). Madrid: Alianza Editorial.

Cornejo, R.; Redondo, J. M. (2001). El Clima escolar percibido por los alumnos de enseñanza media: Una investigación en algunos liceos de la Región Metropolitana. Última década, 9(15),11-52.

Ministerio de Educación de la Nación. (2018). Aprender 2017. Informe de resultados. Secundaria. Recuperado en 1 mar. 2019, de https://www.argentina.gob.ar/sites/default/ files/reporte_nacional_2017_secundaria_0.pdf
Moos, R. H.; Tricket, E.J. (1974) Classroom Environment Scale Manual. Palo Alto, California: Consulting Psychologists Press.

Norton, M. S. (2008). Human resource administration for educational leaders. New York: Sage.

Paneiva Pompa, J. P.; Bakker, L.; Rubiales, J. (2018). Estado del conocimiento del Clima Áulico. Características socioemocionales del contexto de enseñanza y aprendizaje. Revista Educación y ciencia Educación y Ciencia, 7(49), 55-64.

Pardo, A.; Ruiz, M. (2000). Spss 11 guía para el análisis de datos. Análisis no paramétrico. México, D. F.: Mc Graw Hill.

Pianta, R. C.; La Paro, K. M.; Payne, C.; Cox, M. J.; Bradley, R. (2002). The relation of kindergarten classroom environment to teacher, family, and school characteristics and child outcomes. The elementary school journal, 102(3), 225-238.

Quezada, C. (2007). Potencia estadística, sensibilidad y Tamaño de efecto: ¿un nuevo canon para la investigación? Onomázein, 16, 159-170. http://www.redalyc.org/ html/1345/134516684004/

Reid, L. D.; Radhakrishnan, P. (2003). Race matters: The relation between race and general campus Climate. Cultural Diversity and Ethnic Minority Psychology, 9(3), 263-275.

Rivera Arrieta, E. E.; Usta González, W. I. (2018). Clima Social Escolar de estudiantes de la institución educativa dolores Garrido de González, Córdoba- Colombia. Tesis para obtener grado de magíster, Universidad Metropolitana de Educación, Ciencia y Tecnología, Panamá. Recuperado en 1 nov. 2018, de http://repositorio.umecit.edu.pa/ handle/001/837

Sternberg, R. J.; Wagner, R. K.; Williams, W. M.; Horvath, J. A. (1995). Testing common sense. American psychologist, 50(11), 912.

Treviño, E.; Valdés, H.; Castro, M.; Costilla, R.; Pardo, C.; Donoso Rivas, F. (2010). Factores asociados al logro cognitivo de los estudiantes de América Latina y el Caribe. OREALC/ UNESCO.

Triano Quijano, A. F.; Velázquez Niño, A. M. (2014). Comunicación asertiva de los docentes y Clima emocional del aula en preescolar. Revista Latinoamericana de Educación, 5(1), 23-41.

Recibido: 29 de marzo de 2019.

Aprobado: 10 de marzo de 2020. 